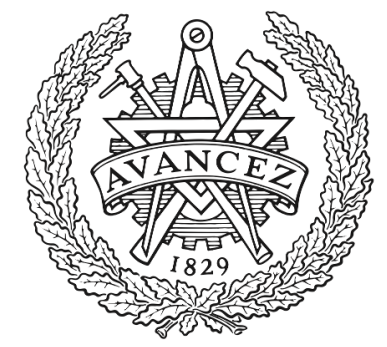

CHALMERS

UNIVERSITY OF TECHNOLOGY

\title{
Manipulation of exciton and trion quasiparticles in monolayer WS2 via charge transfer
}

Downloaded from: https://research.chalmers.se, 2023-04-26 12:37 UTC

Citation for the original published paper (version of record):

Gaur, A., Rivera, A., Dash, S. et al (2019). Manipulation of exciton and trion quasiparticles in monolayer WS2 via charge transfer. Applied Physics Letters, 115(17).

http://dx.doi.org/10.1063/1.5113775

N.B. When citing this work, cite the original published paper. 


\title{
Manipulation of exciton and trion quasiparticles in monolayer $\mathrm{WS}_{2}$ via charge transfer
}

\author{
Cite as: Appl. Phys. Lett. 115, 173103 (2019); doi: 10.1063/1.5113775 \\ Submitted: 8 June 2019 . Accepted: 5 October 2019 . \\ Published Online: 22 October 2019

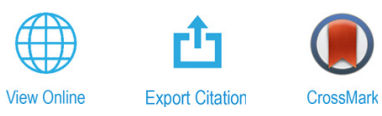

Anand P. S. Gaur, ${ }^{1,2, a)}$ Adriana M. Rivera, ${ }^{7}$ Saroj P. Dash, ${ }^{3}$ Sandwip Dey, ${ }^{4}$ Ram S. Katiyar, ${ }^{7}$ and Satyaprakash Sahoo ${ }^{5,6, a)}$

\author{
AFFILIATIONS \\ 'Department of Physics and Institute for Functional Nanomaterials, University of Puerto Rico, San Juan, Puerto Rico O0931 USA \\ ${ }^{2}$ Department of Materials Science and Engineering, lowa State University, Ames, lowa 50011, USA \\ ${ }^{3}$ Department of Microtechnology and Nanoscience, Chalmers University of Technology, SE-41296 Göteborg, Sweden \\ ${ }^{4}$ Materials Science and Engineering in the School for Engineering of Matter, Transport, and Energy, Arizona State University, \\ Tempe, Arizona 85287, USA \\ ${ }^{5}$ Institute of Physics, Bhubaneswar 751005, India \\ ${ }^{6}$ Homi Bhabha National Institute, Anushaktinagar, Mumbai 400 085, India
}

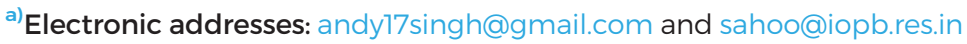

\begin{abstract}
Charge doping in transition metal dichalcogenide is currently a subject of high importance for future electronic and optoelectronic applications. Here, we demonstrate chemical doping in the CVD-grown monolayer (1L) of $\mathrm{WS}_{2}$ by a few commonly used laboratory solvents by investigating the room temperature photoluminescence (PL). The appearance of distinct trionic emission in the PL spectra and quenched $\mathrm{PL}$ intensities suggests $n$-type doping in $\mathrm{WS}_{2}$. The temperature-dependent PL spectra of the doped $1 \mathrm{~L}-\mathrm{WS}_{2}$ reveal a significant enhancement of trions emission intensity over the excitonic emission at low temperature, indicating the stability of trion at low temperature. The temperature-dependent exciton-trion population dynamic has been modeled using the law of mass action of trion formation. These results shed light on the solution-based chemical doping in $1 \mathrm{~L}-\mathrm{WS}_{2}$ and its profound effect on the photoluminescence which is essential for the control of optical and electrical properties for optoelectronic applications.
\end{abstract}

Published under license by AIP Publishing. https://doi.org/10.1063/1.5113775

Several two dimensional (2D) materials beyond graphene have emerged rapidly, showing remarkable physical properties. ${ }^{1,2}$ Among these, the semiconducting transition metal dichalcogenides (TMDs), such as $\mathrm{MoS}_{2}, \mathrm{WS}_{2}$, and $\mathrm{WSe}_{2}$, stood out as phenomenal materials owing to the evolution of direct optical bandgap, optically addressable spin-polarized valleys ( $\pm \mathrm{K}$ points), and wide spin-orbit splitting. ${ }^{4-6}$ These are the added functionalities in their monolayer architecture and could be explored further in proposed novel valleytronics, spintronic, and optoelectronic device applications ${ }^{7-9}$ along with the demonstrated capability as the excellent channel layer in the planar FET devices. ${ }^{3,10}$ The other fascinating aspect of the monolayer TMDs is that these provide a great framework to study the fundamental manybody problems of condensed matter physics. It has been emphasized that the strong Coulombic interaction among the charged particles yields multiparticle complexes such as excitons, trions (charged excitons), and biexcitons. ${ }^{11-13}$ Extreme confinement and strong interacting forces lead further to the creation of the higher order correlated states such as exciton condensates and dropletons. It is noteworthy that the magnitude of the binding energy (BE) of these many-body systems is extensively massive compared to other sub-nano-dimensional materials (quantum dots and nanowires) providing room temperature stability to these particles. ${ }^{14,15}$ The concentration/density of such many-body systems could have a direct impact in modifying the physical properties under specific conditions.

For example, recently, the electrical transport measurement performed on 1L-TMDs FET device with simultaneous measurements of photoluminescence $(\mathrm{PL})^{16}$ demonstrated the distinguishable bright trionic emission with a noticeable anomalous negative photoconductivity at room temperature under the gate bias voltage. ${ }^{17,18}$ The negative photoconductivity assigned to an increased carrier effective mass relevant to the formation of trions. ${ }^{18}$ Similarly, an inhomogeneity observed in the PL intensity map of $1 \mathrm{~L}-\mathrm{WS}_{2}$ and $\mathrm{MoS}_{2}$ was ascribed to the chemical inhomogeneity and the presence of defects. ${ }^{19,20}$ These results suggested that at the ultrathin scale, the physical properties of single layer TMDs are responsive to its surrounding stimulus, implicating that the electronic and optical properties are tunable through 
surface modifications. In an alternative approach, a high trion density was achieved through the surface charge transfer doping via the chemical treatment of $1 \mathrm{~L}-$ TMDs. ${ }^{21,22}$ This is an emerging research area dedicated to modifying the electronic properties in various nanomaterials by covalent or noncovalent functionalization, and particularly helpful to understand the nonlinear optical dynamical properties and device characteristics.

$1 \mathrm{~L}-\mathrm{WS}_{2}$ is isostructural to $1 \mathrm{H}-\mathrm{MoS}_{2}$ and is well known for its high quantum yield of $\sim 6 \%$, producing giant $\mathrm{PL}$ at room temperature, ${ }^{20,23}$ evolving from a single state decay in contrast to a multiple state decay as observed in $\mathrm{MoS}_{2}$. Although the contribution of other levels, i.e., trap states generated by defects, surface states are feeble in high-quality $1 \mathrm{~L}-\mathrm{WS}_{2}$. However, a significant change in the PL spectrum has been reported in the CVD-grown $1 \mathrm{~L}-\mathrm{WS}_{2}$ after charge transfer through noncovalent functionalization by an organic molecule. ${ }^{24}$ In this report, we further investigated the surface functionalization of $1 \mathrm{~L}-\mathrm{WS}_{2}$ by dielectric solvents and studied the effect of physisorption of these solvents on the optical properties. The optical properties are investigated using the PL and micro-Raman scattering experiments. Further, the temperature-dependent PL sheds light on the excitontrion dynamics and establishes the fact that $n$-type charge doping occurs by polar solvents (e.g., chemical doping), and these results are modeled by solving the law of mass action for trion formation in corroborating the temperature-dependent findings.

The details of the synthesis of monolayer $1 \mathrm{~L}-\mathrm{WS}_{2}$ on the $\mathrm{Al}_{2} \mathrm{O}_{3}$ substrate by the chemical vapor deposition method are reported elsewhere. ${ }^{27}$ The morphology of the CVD-grown $1 \mathrm{~L}-\mathrm{WS}_{2}$ on large area $\mathrm{Al}_{2} \mathrm{O}_{3}$ substrate was observed using the JEOL scanning electron microscopy and the VEECO atomic force microscopy (AFM) systems. The monolayer nature of the samples was characterized using microRaman and PL spectroscopies. Raman scattering was performed using a Horiba T64000 spectrometer, the $514.5 \mathrm{~nm}$ line of the Ar ion laser was used as the excitation source (with the sample exposed to a 0.5 $\mathrm{mW}$ laser power) and an $80 \times$ objective was used to focus the laser on the sample. The PL experiments were performed using the same setup.

The SEM image of the as-deposited $1 \mathrm{~L}-\mathrm{WS}_{2}$ on the $\mathrm{Al}_{2} \mathrm{O}_{3}$ substrate is illustrated in Fig. 1(a), showing the typically equilateral triangular pattern with large uneven ragged edges with dimensions of $\sim 50$ $\mu \mathrm{m}$. Recently, Zhang et al. ${ }^{25}$ reported similar features in the CVDgrown $1 \mathrm{~L}-\mathrm{WS}_{2}$ and proposed that the high-temperature CVD synthesis and the use of argon carrier gas favor the considerable $1 \mathrm{~L}-\mathrm{WS}_{2}$ size deposition with crooked edges.

The uneven edges also proved to contain a zig-zag sulfur arrangement, whereas the sharp edge contains Mo atoms. ${ }^{26}$ Moreover, the measured thickness of $1 \mathrm{~L}-\mathrm{WS}_{2}$, determined by the atomic force

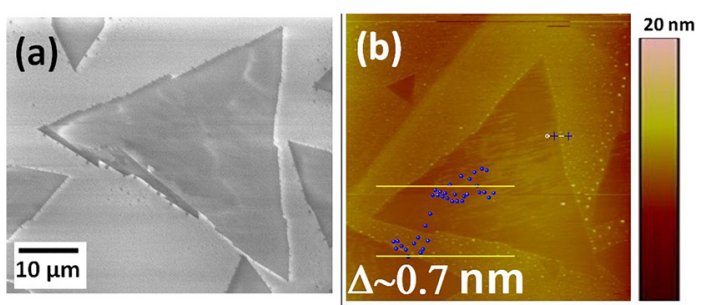

FIG. 1. (a) SEM and (b) AFM images of $1 \mathrm{~L}-\mathrm{WS}_{2}$. The CVD-grown $\mathrm{WS}_{2}$ has a size of $50 \mu \mathrm{m}$ and a thickness of $0.7 \mathrm{~nm}$. microscopy (AFM) shown in Fig. 1(b), was found in a close agreement to monolayer thickness. The as-deposited $1 \mathrm{~L}-\mathrm{WS}_{2}$ was further characterized by optical and Raman scattering experiments; the latter technique was employed routinely to determine the different sets of physical properties in the majority of the layered materials. ${ }^{27-30} \mathrm{~A}$ comparison of the Raman spectra of the CVD-grown $1 \mathrm{~L}-\mathrm{WS}_{2}$ with its bulk reference is shown in Fig. 2(a). The red shift of the $A_{1 g}$ mode by $2.3 \mathrm{~cm}^{-1}$ and the blue shift of the $\mathrm{E}_{2 \mathrm{~g}}^{1}$ mode along with the evolution of the zone edge longitudinal acoustic phonon mode (at $\mathrm{M}$ point of Brillouin zone) longitudinal acoustic (2LA) mode further corroborated the SEM and AFM measurements.

The evolution of the direct bandgap and the extreme charge confinement in a single layer sheet rendered the emanation of a giant PL at room temperature in $1 \mathrm{~L}-\mathrm{WS}_{2}$. In Fig. 2(b), a comparison of a room temperature PL spectrum is made between an as-grown $1 \mathrm{~L}-\mathrm{WS}_{2}$, bilayer, and bulk $\mathrm{WS}_{2}$. As anticipated, the as-grown $1 \mathrm{~L}-\mathrm{WS}_{2}$ sample showed a giant PL intensity over the bilayer and bulk $\mathrm{WS}_{2}$. The PL band was fitted appropriately with two Lorentzian functions: an intense peak and a weak peak at 2.01 and $1.98 \mathrm{eV}$, respectively, as shown in Fig. 2(c). The intense peak is assigned to radiative transition arising from the free exciton recombination, while the weak peak is related to the trion recombination. ${ }^{31}$ The presence of the trionic recombination also explains the left skewness of the PL band which has been regularly reported in the CVD-grown $1 \mathrm{~L}-\mathrm{WS}_{2}$. Further, we employed the micro-PL mapping to record the intensity distribution in $1 \mathrm{~L}-\mathrm{WS}_{2}$. As shown in Fig. 2(d) the maximum PL intensity was found in the center of the triangule and reduced gradually toward the edges. To further understand this variation in the PL intensity, the PL spectrum was recorded at different spots within the $1 \mathrm{~L}-\mathrm{WS}_{2}$ [marked as 1, 2, and 3 in Fig. 2(d)]. The PL bandwidth corresponding to the brightest area (spot 1) was found to be relatively narrow (see the supplementary material Fig. S1) and showed successive broadening for
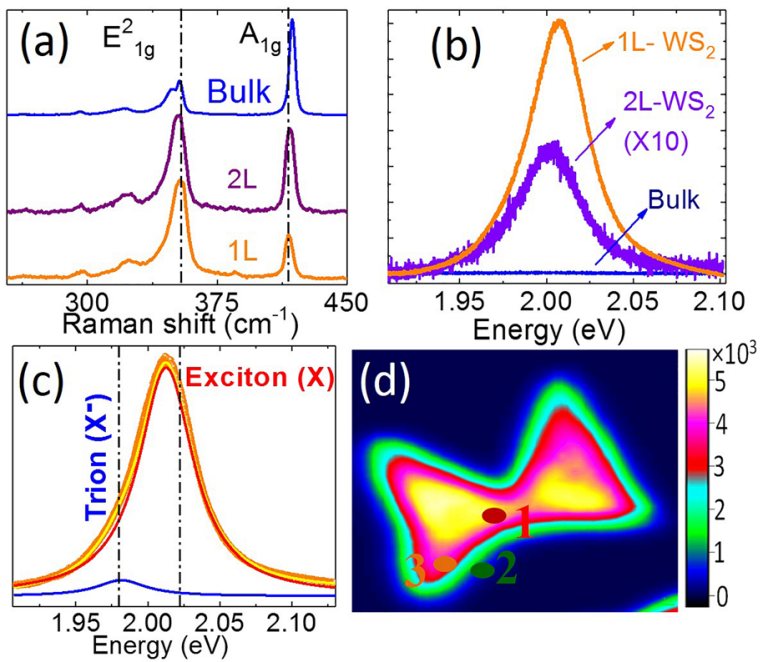

FIG. 2. Optical characterizations of $1 \mathrm{~L}-W S_{2}$ (a) comparison of Raman spectra of $1 \mathrm{~L}, 2 \mathrm{~L}$, and bulk $\mathrm{WS}_{2}$. (b) Room temperature photoluminescence of $1 \mathrm{~L}, 2 \mathrm{~L}$, and bulk $\mathrm{WS}_{2}$. (c) The PL band is fitted with two peaks, and the low energy and the high energy peaks are denoted as trionic $\left(\mathrm{X}^{-}\right)$and excitonic $(\mathrm{X})$ emissions, respectively. (d) Photoluminescence mapping image of two triangular $1 \mathrm{~L}-\mathrm{WS}_{2}$ sharing the corner. 


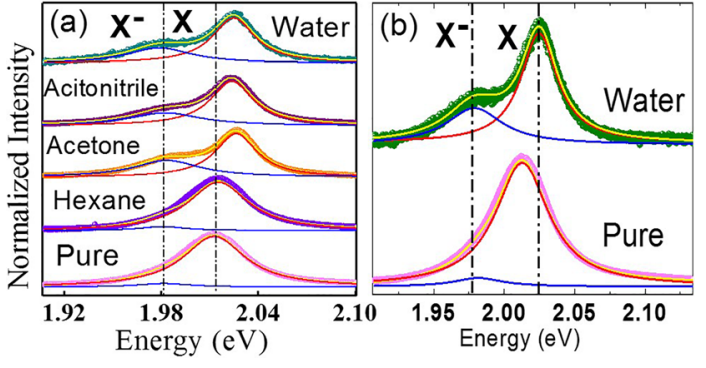

FIG. 3. (a) Room temperature photoluminescence spectra of pure, hexane, acetone, acetonitrile, and water (sequenced from bottom to top spectra) treated $1 \mathrm{~L}-$ $W_{2}$. Spectra are fitted with two peaks for exciton $(X)$ and trion $\left(X^{-}\right)$. (b) For clarity, the pure and water treated spectra are shown.

the other two regions corresponding to spots 2 and 3, respectively. Previously, the broadening in the PL band was described by the formation of bound excitons facilitated by the presence of atomic vacancies. Since the energies of bound excitons are slightly lower than that of free excitons their presence causes a substantial broadening and skewness in the PL spectra. Therefore, the observed PL intensity map suggests a high crystallinity in the central region, but relatively higher defect density toward the edges mitigating the PL intensity through the trion formation.

In our previous work, we probed the polarity of defect states through the chemical doping method in the CVD-grown $1 \mathrm{~L}-\mathrm{WS}_{2}{ }^{32}$ The ease of maneuvering of the trion density and hence their spectral density through surface charge doping could be extended to sense the liquids/vapors used as solvents in the laboratory (such as hexane, acetone, acetonitrile, and water) optically using PL as the optical probe. Thus, we carried out room temperature PL measurements on the chemically treated $1 \mathrm{~L}-\mathrm{WS}_{2}$. The PL spectrum, recorded in vacuum at room temperature, of as-prepared, hexane, acetone, and acetonitrile treated $1 \mathrm{~L}-\mathrm{WS}_{2}$ are represented in Fig. 3(a). Note that the $1 \mathrm{~L}-\mathrm{WS}_{2}$ did not show any significant erosion upon chemical treatment. The PL spectrum of $1 \mathrm{~L}-\mathrm{WS}_{2}$ showed a considerable rise in the trion spectral density for polar solvent treatment; however, no such change was observed in hexane treated $1 \mathrm{~L}-\mathrm{WS}_{2}$.

Before recording the PL spectrum, the nature of the doping in chemically treated $1 \mathrm{~L}-\mathrm{WS}_{2}$ was determined by Raman scan (see the supplementary material Fig. S2). The red shift in $A_{1 g}$ optical mode is indicative of the n-type doping ${ }^{33}$ in treated $1 \mathrm{~L}-\mathrm{WS}_{2}$, which could be further corroborated by the fact that all the used solvents are categorized as Lewis base (lone pair of an electron donor), except hexane. Thus, the observed decrease in the PL intensities in the present study is the result of the $n$-type doping provided by polar solvents in $1 \mathrm{~L}$ -
$\mathrm{WS}_{2}$. The noted spectral feature in the PL spectrum was fitted with two peaks ( $\mathrm{X}$ and $\mathrm{X}^{-}$); the peak positions, full width at half maximum (FWHM), and the intensity ratio of the $\mathrm{X}$ and $\mathrm{X}^{-}$emissions, as summarized in Table I. For more clarity, the fitted PL spectrum of asgrown and water treated $1 \mathrm{~L}-\mathrm{WS}_{2}$ are provided separately in Fig. 2(b). As indicated in Table I, in chemically treated $1 \mathrm{~L}-\mathrm{WS}_{2}$, the most intense peak due to the excitonic emission became narrower and blue shifted by $0.01 \mathrm{eV}$ with the substantial increase in the PL intensity of the trionic emission process. It is also worth noting that the PL intensities of the polar liquid treated samples were substantially quenched (4-6 times). Several external factors, including temperature, mechanical strain, dielectric screening, and doping, could influence the photoinduced electron transition energies in 1L-TMDs.

Thus, to avoid the heating and strain induced PL changes, the laser power density was kept sufficiently low, and the samples were dried at an ambient temperature for several hours prior to the experiment. The analysis of the fitted PL peaks, summarized in Table I, also highlights a few other critical points. Such as, the FWHM of the trionic peak remains more or less unchanged irrespective of the solvents used, but the width of the free exciton peak reduces considerably (almost 40-50\%), which could be due to the inherent defects "metal/chalcogen vacancies and charged surface states" present in the CVD-grown 1L$\mathrm{WS}_{2}$. These defects were intended to bind with the free excitons to form bound excitons rather than trions. ${ }^{34}$ The energy of these bound excitons is even lower than that of the trions, contributing to the asymmetrical shape of the PL band at the lower energy side. When treated with polar solvents, most of the defect sites and charged states interact strongly with physisorbed molecules. In particular, the annihilation of surface states by polar solvents decreases the population of such defect states. Consequently, the FWHM of the exciton peak, which primarily represents the free exciton, narrows down. This assumption is further facilitated by the fact that no such noticeable changes either in the peak position or in the FWHM of the excitonic peak were observed when nonpolar hexane is used. This shows that hexane perhaps does not interact strongly with defects or surface states of $1 \mathrm{~L}-\mathrm{WS}_{2}$; therefore, the population density of the bound exciton is least affected.

However, the variation in the density of free exciton and trion is reflected in the exciton to trion peak intensity ratio $\left(\mathrm{I}_{\mathrm{x}} / \mathrm{I}_{\mathrm{x}}-\right)$. As per Table I, this ratio is highest (almost 10) for pristine and hexane treated $1 \mathrm{~L}-\mathrm{WS}_{2}$, whereas it drops significantly in polar solvent treated $1 \mathrm{~L}$ $\mathrm{WS}_{2}$. It is also noticed that the PL intensities decrease when treated with polar solvents followed by the drying of the samples, which suggests that the effect of dielectric screening ${ }^{35}$ may not account for the profound change observed in our PL studies. Therefore, to qualitatively explain the obtained PL results, the focus will be on charge doping in $1 \mathrm{~L}-\mathrm{WS}_{2}$ and its effect on the PL spectra.

TABLE I. The analyzed PL peak position and FWHM of chemically treated $\mathrm{WS}_{2}$.

\begin{tabular}{lcccccc}
\hline \hline Sample & Position of X $(\mathrm{eV})$ & Position of $\mathrm{X}^{-}(\mathrm{eV})$ & Intensity ratio X/ X & FWHM, X & FWHM, $^{-}$ & $\mathrm{X}^{-} \mathrm{BE}(\mathrm{meV})$ \\
\hline Pure & 2.013 & 1.981 & 10.5 & 0.055 & 0.047 & $\sim 32$ \\
Hexane & 2.015 & 1.981 & 7.2 & 0.050 & 0.047 & $\sim 34$ \\
Acetone & 2.024 & 1.981 & 1.6 & 0.029 & 0.054 & $\sim 43$ \\
Acetonitrile & 2.022 & 1.980 & 2 & 0.032 & 0.053 & $\sim 42$ \\
Water & 2.025 & 1.977 & 1.9 & 0.031 & 0.048 & $\sim 48$ \\
\hline \hline
\end{tabular}


Additionally, the magnitude of this ratio is different for all polar liquids, indicating that the level of doping induced by each solvent is different. Moreover, the experimentally observed blue shift of the exciton peak and the red shift of the trion peak are the consequence of the renormalization of the energy bands for exciton and trion and explained under the combined effect of Pauli blocking and many-body interaction. ${ }^{12,36}$ However, the observed changes in the PL behavior can be qualitatively addressed by considering the interplay between the exciton and trion dynamics model comprising the exciton, trion, and ground states. The photogenerated excitons undergo two possible radiative decay transition channels; the excited electron can combine directly with the hole or it can form a trion. In the real sense, it is the decay rate of the exciton (with or without trion formation) that eventually determine spectral weights.

The spectral display of both exciton and trion is expected to exhibit some fine characteristics at liquid nitrogen temperature, as has been observed consistently in 1L-TMDs. ${ }^{17}$ Since water and acetonitrile treated $1 \mathrm{~L}-\mathrm{WS}_{2}$ showed a substantial change in their room temperature PL spectrum a temperature-dependent PL measurement was carried out in these chemically treated samples. Figures 4(a) and 4(b) show the temperature-dependent PL spectra of water and acetonitrile treated 1L$\mathrm{WS}_{2}$, respectively. Evidently, the low-temperature spectral characteristics are quite different from the pristine sample (see the supplementary material Fig. S3). At liquid nitrogen temperature, the PL signature of the free excitonic emission was thoroughly subdued; however, a trionic emission enhanced substantially along with the emergence of an extra PL shoulder [marked as an asterisk in both Figs. 4(a) and 4(b)] below $150 \mathrm{~K}$. This PL shoulder is assigned to defect bound localized excitons. These localized states are thermally unstable due to their low binding energy $(\sim 20 \mathrm{meV})$ and dissociate easily because of thermal fluctuations even at room temperature. As evident from Figs. 4(a) and 4(b), the spectral density of the defect bound exciton is closer to the trion spectral density in acetonitrile treated $1 \mathrm{~L}-\mathrm{WS}_{2}$ compared to water treated $1 \mathrm{~L}-$ $\mathrm{WS}_{2}$. This aligns very well with the fact that in water treated $1 \mathrm{~L}-\mathrm{WS}_{2}$ defect/surface states neutralized significantly over acetonitrile treated
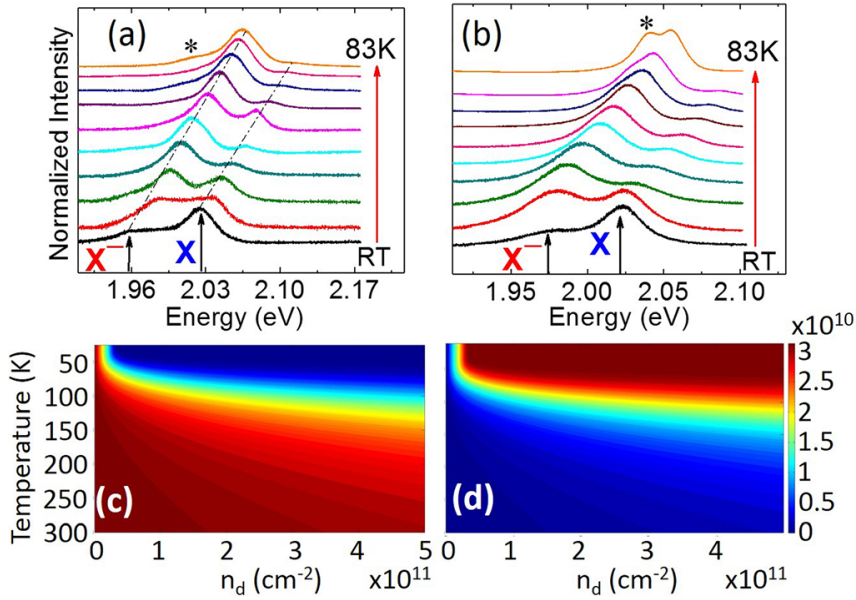

FIG. 4. (a) and (b) Temperature-dependent photoluminescence of water and acetonitrile treated $1 \mathrm{~L}-\mathrm{WS}_{2}$, respectively, and (c) and (d) plots of the variation of exciton and trion concentrations as a function of temperature and doping concentration obtained by solving the law of mass action model for trion.
$1 \mathrm{~L}-\mathrm{WS}_{2}$; hence, the PL intensity of the defect bound exciton is lower in water than that in acetonitrile treated $1 \mathrm{~L}-\mathrm{WS}_{2}$ monolayer.

The temperature-dependent exciton and the trion peak positions for water treated samples were analyzed by fitting the spectra to the Lorentzian function, and the results are plotted in Fig. S4 of the supplementary material. The temperature dependences fit well with the O'Donnell equation $\left\{\mathrm{E}_{\mathrm{g}}(T)=\mathrm{E}_{\mathrm{g}}(0)-S\langle\hbar \omega\rangle[\operatorname{coth}(\langle\hbar \omega\rangle) /(2 k T)-1]\right\}$ that accounts for electron-phonon interaction $S$, and the trion binding energy is found to be independent of temperature. ${ }^{37}$ Assuming that the spectral weights of exciton and trion at a given temperature are proportional to their concentration, we evaluated the temperature dependency of the spectral weight of trions and excitons by considering a steady state model. ${ }^{38}$ This model considers the (i) concentration of photoexcited electron $\left(n_{p}\right)$ to be the sum total of the concentration of exciton $\left(N_{X}\right)$ and trion $\left(N_{X^{-}}\right)$, i.e., $n_{p}=N_{X}+N_{X}-$ and (ii) the concentration of $n$ doping in the absence of light $\left(n_{d}\right)$ to be the sum total of the concentration of free electron $\left(n_{e}\right)$ and trion $\left(N_{X}-\right)$, i.e., $n_{d}=n_{e}+N_{X}-$. The law of mass action for trion formation $\left(\mathrm{X}+\mathrm{e}^{-} \rightarrow \mathrm{X}^{-}\right)$is used to derive the relationship between the quantities mentioned above as follows:

$$
\frac{N_{X} n_{e}}{N_{X^{-}}}=\frac{4 m_{X} m_{e}}{\pi \hbar^{2} m_{X^{-}}} k_{B} T \exp { }^{\left(\frac{-E_{b}}{k_{B} T}\right)}
$$

where $m_{X}, m_{X^{-}}$, and $m_{e}$ are the effective mass of exciton, trion, and electron, respectively, and $E_{b}$ is the trion binding energy. By solving these equations, one can get the following expression for trion and exciton concentrations:

$$
\begin{gathered}
N_{X^{-}}=\frac{n_{p}+n_{d}+n_{A}-\sqrt{\left(n_{p}+n_{d}+n_{A}\right)^{2}-4 n_{p} n_{d}}}{2}, \\
N_{X}=\frac{n_{A} N_{X^{-}}}{n_{d}-N_{X^{-}}},
\end{gathered}
$$

where $n_{A}=A k_{B} T \exp \left(-E_{b} / K_{B} T\right)$. The variations of the concentration of exciton and trion as a function of temperature and doping are plotted in Figs. 4(c) and 4(d), respectively. At a very low doping, the simulation shows that excitons are predominant over trions at room temperature and with increase in doping the concentration of exciton and trion decreases and increases, respectively. From our experiment, at room temperature, we obtain a minimum value for the intensity ratio of exciton and trion to be about 1.6 (from Table I), and to get such an intensity ratio, the simulation suggests a high doping concentration caused by polar solvents. Furthermore, the simulation indicates that for a given doping concentration, with the decrease in temperature, the exciton and trion concentrations go down and up, respectively. This is consistent with our low temperature PL results. We also demonstrated by laser heating that the effect of polar solvents on the room temperature PL of monolayer $1 \mathrm{~L}-\mathrm{WS}_{2}$ is reversible (see the supplementary material Fig. S5).

In summary, the PL spectra of $1 \mathrm{~L}-\mathrm{WS}_{2}$ grown via chemical vapor deposition were analyzed by maneuvering the interplay among free exciton, bound exciton, and trion concentrations through the polar solvent treatment. The polar solvent introduced excess negative charge (n-type doping) through the surface charge transfer by adsorbed molecules, resulting in a substantial increase in the trionic spectral weight, as observed in the PL spectrum of chemically treated $1 \mathrm{~L}-\mathrm{WS}_{2}$. Besides, the FWHM of the free exciton PL band became narrower, leading to the fact that defect/surface states were suppressed significantly after 
the chemical treatment. The negative electron doping was confirmed by Raman and PL scans, respectively. Further, in the temperaturedependent PL spectrum, an extra feature associated with bound exciton along with the trion emission evolved at the expense of the free exciton emission at the lowest temperature. Such an uncommon PL behavior is seldom observed in the CVD-grown $1 \mathrm{~L}-\mathrm{WS}_{2}$. The temperature-dependent behavior of excitonic and trionic peaks was simulated by a model using the law of mass action for trion formation. These results shed light on the fundamental understanding of the electronic properties and also extend a method to sense the reactive gases optically.

See the supplementary material for the PL spectra of $1 \mathrm{~L}-\mathrm{WS}_{2}$; position and laser power, plot of temperature-dependent exciton, and trion position. Temperature-dependent as-prepared $1 \mathrm{~L}-\mathrm{WS}_{2}$. Raman spectra of acetonitrile and water treated $1 \mathrm{~L}-\mathrm{WS}_{2}$.

A.P.S.G., A.M.R., and R.S.K. acknowledge the financial support from DOE (Grant No. DEG02-ER46526).

\section{REFERENCES}

1J. Wang, S. Deng, Z. Liu, and Z. Liu, Natl. Sci. Rev. 2, 22 (2015).

${ }^{2}$ K. Ang, Z. Ling, and J. Zhu, in 2015 IEEE International Conference on Digital Signal Processing (2015), pp. 1223-1226.

${ }^{3}$ K. F. Mak, C. Lee, J. Hone, J. Shan, and T. F. Heinz, Phys. Rev. Lett. 105, 136805 (2010).

${ }^{4}$ F. Xia, H. Wang, D. Xiao, M. Dubey, and A. Ramasubramaniam, Nat. Photonics 8, 899 (2014).

${ }^{5}$ K. F. Mak and J. Shan, Nat. Photonics 10, 216 (2016).

${ }^{6}$ Q. H. Wang, K. Kalantar-Zadeh, A. Kis, J. N. Coleman, and M. S. Strano, Nat. Nanotechnol. 7, 699 (2012).

${ }^{7}$ D. Jariwala, V. K. Sangwan, L. J. Lauhon, T. J. Marks, and M. C. Hersam, ACS Nano 8, 1102 (2014).

${ }^{8}$ K. F. Mak, K. He, J. Shan, and T. F. Heinz, Nat. Nanotechnol. 7, 494 (2012).

${ }^{9}$ A. Dankert, P. Pashaei, M. V. Kamalakar, A. P. S. Gaur, S. Sahoo, I. Rungger, A. Narayan, K. Dolui, M. A. Hoque, R. S. Patel, M. P. De Jong, R. S. Katiyar, S. Sanvito, and S. P. Dash, ACS Nano 11, 6389 (2017).

${ }^{10}$ B. Radisavljevic, A. Radenovic, J. Brivio, V. Giacometti, and A. Kis, Nat. Nanotechnol. 6, 147 (2011).

${ }^{11}$ H. Shi, H. Pan, Y.-W. Zhang, and B. I. Yakobson, Phys. Rev. B 87, 155304 (2013).

${ }^{12}$ T. Ogawa, J. Phys.: Condens. Matter 16, S3567 (2004).

${ }^{13}$ M. Barbone, A. R.-P. Montblanch, D. M. Kara, C. Palacios-Berraquero, A. R. Cadore, D. De Fazio, B. Pingault, E. Mostaani, H. Li, B. Chen, K. Watanabe, T. Taniguchi, S. Tongay, G. Wang, A. C. Ferrari, and M. Atatüre, Nat. Commun. 9, 3721 (2018).
${ }^{14}$ K. F. Mak, K. He, C. Lee, G. H. Lee, J. Hone, T. F. Heinz, and J. Shan, Nat. Mater. 12, 207 (2012).

${ }^{15}$ A. Chernikov, T. C. Berkelbach, H. M. Hill, A. Rigosi, Y. Li, O. B. Aslan, D. R. Reichman, M. S. Hybertsen, and T. F. Heinz, Phys. Rev. Lett. 113, 76802 (2014).

${ }^{16}$ B. Zhu, X. Chen, and X. Cui, Sci. Rep. 5, 9218 (2015).

${ }^{17}$ J. S. Ross, S. Wu, H. Yu, N. J. Ghimire, A. M. Jones, G. Aivazian, J. Yan, D. G. Mandrus, D. Xiao, W. Yao, and X. Xu, Nat. Commun. 4, 1474 (2013).

${ }^{18}$ C. H. Lui, A. J. Frenzel, D. V. Pilon, Y.-H. Lee, X. Ling, G. M. Akselrod, J. Kong, and N. Gedik, Phys. Rev. Lett. 113, 166801 (2014).

${ }^{19}$ S. Tongay, J. Suh, C. Ataca, W. Fan, A. Luce, J. S. Kang, J. Liu, C. Ko, R. Raghunathanan, J. Zhou, F. Ogletree, J. Li, J. C. Grossman, and J. Wu, Sci. Rep. 3, 2657 (2013).

${ }^{20}$ H. R. Gutiérrez, N. Perea-López, A. L. Elías, A. Berkdemir, B. Wang, R. Lv, F. López-Urías, V. H. Crespi, H. Terrones, and M. Terrones, Nano Lett. 13, 3447 (2013).

${ }^{21}$ J. D. Lin, C. Han, F. Wang, R. Wang, D. Xiang, S. Qin, X.-A. Zhang, L. Wang, H. Zhang, A. T. S. Wee, and W. Chen, ACS Nano 8, 5323 (2014).

${ }^{22}$ D. Kiriya, M. Tosun, P. Zhao, J. S. Kang, and A. Javey, J. Am. Chem. Soc. 136, 7853 (2014).

${ }^{23}$ L. Yuan and L. Huang, Nanoscale 7, 7402 (2015).

${ }^{24}$ N. Peimyoo, W. Yang, J. Shang, X. Shen, Y. Wang, and T. Yu, ACS Nano 8, 11320 (2014).

${ }^{25}$ Y. Zhang, Y. Zhang, Q. Ji, J. Ju, H. Yuan, J. Shi, T. Gao, D. Ma, M. Liu, Y. Chen, X. Song, H. Y. Hwang, Y. Cui, and Z. Liu, ACS Nano 7, 8963 (2013).

${ }^{26}$ A. M. van der Zande, P. Y. Huang, D. A. Chenet, T. C. Berkelbach, Y. You, G.H. Lee, T. F. Heinz, D. R. Reichman, D. A. Muller, and J. C. Hone, Nat. Mater. 12, 554 (2013).

${ }^{27}$ A. P. S. Gaur, S. Sahoo, J. F. Scott, and R. S. Katiyar, J. Phys. Chem. C 119, 5146 (2015).

${ }^{28}$ A. Berkdemir, H. R. Gutiérrez, A. R. Botello-Méndez, N. Perea-López, A. L. Elías, C.-I. Chia, B. Wang, V. H. Crespi, F. López-Urías, J.-C. Charlier, H. Terrones, and M. Terrones, Sci. Rep. 3, 1755 (2013).

${ }^{29}$ H. Li, Q. Zhang, C. C. R. Yap, B. K. Tay, T. H. T. Edwin, A. Olivier, and D. Baillargeat, Adv. Funct. Mater. 22, 1385 (2012).

${ }^{30}$ S. Sahoo, A. P. S. Gaur, M. Ahmadi, M. J.-F. Guinel, and R. S. Katiyar, J. Phys. Chem. C 117, 9042 (2013).

${ }^{31}$ Y. Kobayashi, S. Sasaki, S. Mori, H. Hibino, Z. Liu, K. Watanabe, T. Taniguchi, K. Suenaga, Y. Maniwa, and Y. Miyata, ACS Nano 9, 4056 (2015).

${ }^{32}$ A. M. Rivera, A. P. S. Gaur, S. Sahoo, and R. S. Katiyar, J. Appl. Phys. 120, 105102 (2016).

${ }^{33}$ B. Chakraborty, A. Bera, D. V. S. Muthu, S. Bhowmick, U. V. Waghmare, and A. K. Sood, Phys. Rev. B 85, 161403 (2012).

${ }^{34}$ V. Carozo, Y. Wang, K. Fujisawa, B. R. Carvalho, A. McCreary, S. Feng, Z. Lin, C. Zhou, N. Perea-López, A. L. Elías, B. Kabius, V. H. Crespi, and M. Terrones, Sci. Adv. 3, e1602813 (2017).

${ }^{35}$ Y. Lin, X. Ling, L. Yu, S. Huang, A. L. Hsu, Y.-H. Lee, J. Kong, M. S. Dresselhaus, and T. Palacios, Nano Lett. 14, 5569 (2014).

${ }^{36}$ P. Hawrylak, Phys. Rev. B 44, 3821 (1991).

${ }^{37}$ K. P. O’Donnell and X. Chen, Appl. Phys. Lett. 58, 2924 (1991).

${ }^{38}$ S. Mouri, Y. Miyauchi, and K. Matsuda, Nano Lett. 13, 5944 (2013). 